\title{
Osteopontin level and promoter polymorphism in patients with metastatic breast cancer
}

\author{
M.A. Elbaiomy $M D, *$ T. Akl MD, ${ }^{*}$ R. Elhelaly $M D_{,}^{\dagger}$ W. El-Beshbishi MD, ${ }^{\ddagger}$ M.S. El Ghonemy $M D, \$$ \\ and R. Elzehery $M D^{\dagger}$
}

\begin{abstract}
Background Cancer initiation typically occurs when a proto-oncogene's coding region undergoes mutation, resulting in uncontrollable cell growth and division, or when a tumour suppressor gene's coding region is affected by a mutation that inhibits activity of the resulting gene product. The pathophysiologic result is, respectively, exaggerated cell-cycle growth or deficient programmed cell death. Osteopontin (OPN) is an integrin-binding phosphoprotein that is expressed on the surface of normal cells. Osteopontin has a major role in diverse tumour components, especially those implicated in invasion and metastasis. In the present study, we aimed to illustrate the value of oPN as a possible contributor in breast cancer (BCa).
\end{abstract}

Methods This prospective study included 115 patients newly diagnosed with BCa and distant metastasis who were recruited from the Oncology Center, Mansoura University, and the Department of Clinical Oncology and Nuclear Medicine, Mansoura University Hospital, Egypt. The patients recruited had been diagnosed with disseminated visceral metastasis (visceral crisis), with or without bone metastasis; patients with cranial metastasis were excluded from the study. All patients received first-line chemotherapy with docetaxel $75 \mathrm{mg} / \mathrm{m}^{2}$ plus cisplatin $75 \mathrm{mg} / \mathrm{m}^{2}$ or carboplatin 6 AUC (area under the curve) on day 1 every 21 days for a maximum of 6 cycles or till development of toxicity. Trastuzumab (in cases of HER2-positive disease) was given whenever possible (if government assistance or personal finances permitted). Serum levels of OPN were assessed by enzyme-linked immunosorbent assay (ELISA) before treatment was started. A group of 30 matched healthy women whose median serum opN level was $15 \mathrm{ng} / \mathrm{dL}$ were included, and that level was therefore defined as the cut-off value. In addition, oPN gene mutation was determined by polymerase chain reaction (PCR). Correlations of pretreatment serum OPN and OPN gene mutation with various patient clinicopathologic variables, response to the treatment, progression-free survival (PFS), and overall survival (OS) were assessed.

Results Mean serum OPN was highest in HER2-amplified BCa $(64.4 \pm 42.3 \mathrm{ng} / \mathrm{dL})$, and then in triple-negative BCa $(55.9 \pm 34.7 \mathrm{ng} / \mathrm{dL})$, followed by the luminal B and A subtypes $(38.4 \pm 33.1 \mathrm{ng} / \mathrm{dL}$ and $36.3 \pm 32.2 \mathrm{ng} / \mathrm{dL} \mathrm{respectively}$, $p=0.017)$. Testing by PCR revealed that OPN gene mutation was highest in triple-negative BCa ( $85 \%$ OPN mutant vs. $15 \%$ non-mutant), and then in HER2-overexpressed BCa ( $80 \%$ OPN mutant vs. $20 \%$ non-mutant), followed by luminal B BCa (61.9\% OPN mutant vs. $38.1 \%$ non-mutant); the least expression was detected in luminal A BCa (57.9\% OPN mutant vs. $42.1 \%$ non-mutant). Interestingly, patients with high serum OPN and oPN gene mutation experienced both poor PFS (median: 12 months vs. 14 months; $p=0.001$ ) and poor os (median: 14 months vs. 18 months; $p=0.001$ ). Moreover, participants with OPN gene mutation experienced a poor response: of those with progressive disease, $74 \%$ had oPN mutation and $26 \%$ had unmutated oPN $(p=0.04)$. Additionally, high pretreatment serum oPN was correlated with poor treatment response: $49.1 \pm 33.8 \mathrm{ng} / \mathrm{dL}$ in patients with progressive disease and $35.5 \pm 34.3 \mathrm{ng} / \mathrm{dL}$ in those who achieved a complete response, a partial response, or stable disease $(p=0.05)$. Strong concordance was found between high serum OPN and OPN gene mutation in 69 tumours (79.3\%), and strong concordance was detected between normal or low serum OPN and non-mutant OPN in 28 tumours $(60.8 \%)$.

Conclusions The current prospective work helps to highlight oPN as a valid prognostic biomarker for patients with metastatic вСа and reveals that high pretreatment serum OPN and OPN gene mutation are both strongly linked with poor response and survival. Concordance between ELISA and PCR results indicates that either method can be used for the evaluation of OPN. Increased OPN gene mutation in triple-negative BCa could assist in tailoring the treatment response in this very aggressive tumour subtype and could be considered a targetable molecule in future studies.

Key Words Breast cancer, metastatic; osteopontin

Curr Oncol. 2020 October27(5)e444-e450

www.current-oncology.com 


\section{BACKGROUND}

Osteopontin (OPN) is defined as a secreted integrin-binding glycophosphoprotein that expedites cell-matrix interactions and stimulates tumour progression. It is well known that OPN acts through various integrins and CD44, and synergizes with the epidermal growth factor receptor signalling pathway, the hepatocyte growth factor receptor, and Met. In the human body, OPN is expressed in many tissues and cell types; in tumour cells, its expression reaches high levels ${ }^{1}$.

Several signalling pathways, when misregulated, can lead to increased OPN expression. Among them are certain oncogenic and tumour-promoting pathways: receptor tyrosine kinase pathways; $G$ protein pathways; and the Wnt/ $\beta$-catenin, Hedgehog, nuclear factor- $\mathrm{\kappa} B$, and estrogen signalling pathways. On the oPN promoter, several cis-regulatory elements have been identified. One is the Ras-activated enhancer, which binds the Ras-response factor, which is stimulated by Ras signalling in epithelial cells and fibroblasts, resulting in the formation of a complex with Ras-activated enhancer ${ }^{2}$.

For at least $30 \%$ of patients with cancer, the oPN gene is one of the $5 \%$ most highly expressed genes, relative to expression in normal tissue, in 20 of 35 microarray datasets ${ }^{3}$. In RNA microarrays deposited in Oncomine (Thermo Fisher Scientific, Waltham, MA, U.S.A.) and in meta-analysis data, OPN was shown to be associated with reduced apoptosis in bronchogenic carcinoma ${ }^{4}$. Knockdown of oPN in human lung cancer cells has been found to suppress the proliferation and metastasis of lung cancer cells, to trigger autophagy, and to abolish the radioresistance of cancer cells ${ }^{5}$.

Osteopontin modulates the malignant transformation of uterine cancer ${ }^{6}$. Further, opN orchestrates CD44mediated p38 phosphorylation in cervical cancer cells, promoting nuclear factor- $\mathrm{\kappa} \mathrm{B}$-dependent expression of furin, an extracellular protease involved in the processing of human papillomavirus that augments the motility of cervical cancer cells?

Breast cancer (BCa) cells that are oPN-overexpressing demonstrated high anchorage-independent soft agar growth. Those cells also showed higher lymphovascular invasion, lymph node metastasis, and lung micrometastasis, highlighting OPN as a precise molecular player in lymphatic metastasis in $\mathrm{BCa}^{8}$. Additionally, OPN increases both the invasion and metastasis of human mammalian epithelial cells and triggers angiogenesis signalling cascades and vascular endothelial growth factor-dependent tumour growth ${ }^{9}$. Through upregulation of the epidermal growth factor receptor, OPN also regulates many transduction signalling pathways ${ }^{10}$.

\section{METHODS}

The present prospective study was conducted from January 2017 to March 2019, with 115 patients newly diagnosed with metastatic BCa who were recruited from the Oncology Center, Mansoura University, and the Department of Clinical Oncology and Nuclear Medicine, Mansoura University Hospital. All included patients presented with visceral or bone metastasis, or both (visceral crisis). Patients with brain metastasis were excluded, meaning that all participants were candidates for chemotherapy with or without targeted therapy according to their genetic profile and target availability. All patients received first-line chemotherapy [docetaxel $75 \mathrm{mg} / \mathrm{m}^{2}$ plus cisplatin $75 \mathrm{mg} / \mathrm{m}^{2}$ or carboplatin 6 AUC (area under the curve), all on day 1 every 21 days for a maximum of 6 cycles or until the appearance of toxicity). Anti-HER2 therapy (for HER2-positive tumours) was added depending on the availability of financial support.

\section{Analysis of Polymorphisms in the OPN Regulatory Region}

The PCR amplification of the promoter regulatory region from extracted DNA used these primers:

Forward primer 5'-CAAGCTACTGCATACTCGAAATCACA-3'

- Reverse primer 5'-ACAACCAAGCCCTCCCAGAATTTA-3'

The PCR analysis used $50 \mathrm{ng}$ DNA as a template and was processed at $95^{\circ} \mathrm{C}$ for 10 minutes, followed by 36 cycles of $94^{\circ} \mathrm{C}$ for 30 seconds, an annealing temperature for $60 \mathrm{sec}-$ onds, and $72^{\circ} \mathrm{C}$ for 60 seconds, with a final extension at $72^{\circ} \mathrm{C}$ for a total of 15 minutes. A QIAquick Gel Extraction Kit (Qiagen, Carlsbad, CA, U.S.A.) was used for affinity membrane purification, and then PCR products were subjected to cycle sequencing with the aforementioned primers on an automated ABI 310 DNA sequencer (Applied Biosystems, Foster City, CA, U.S.A.).

\section{Measurement of Serum OPN}

Serum opN was measured using an enzyme-linked immunosorbent assay (ELISA) kit supplied by Immuno-Biological Laboratories, Fujioka, Japan. A group of 30 age-matched women without cancer were enrolled; their median serum OPN of $15 \mathrm{ng} / \mathrm{dL}$ was used as a cut-off value.

Correlations of pretreatment serum OPN and OPN gene polymorphism with various patient clinicopathologic criteria, response to treatment, progression-free survival (PFS), and overall survival (OS) were assessed.

\section{Ethics Approval}

All procedures in this study were conducted in accordance with the ethics standards of the institutional and national research committee and with the 1964 Helsinki Declaration and its recent amendments or comparable ethics standards.

\section{RESULTS}

The study included 115 patients with newly diagnosed metastatic BCa, of whom 39 (33.9\%) were premenopausal, and $76(66.1 \%)$, postmenopausal. Age range in the cohort was 31-74 years. Of the 115 patients, 41 (35.7\%) presented with metastases to both viscera and bone; 74 patients $(64.3 \%)$ presented with visceral metastasis only. Mean pretreatment serum OPN was $44.2 \pm 35.5 \mathrm{ng} / \mathrm{dL}$ (range: $5-136 \mathrm{ng} / \mathrm{dL}$ ). By sequencing, 77 patients (67\%) had mutant OPN, and 38 patients $(33 \%)$ had non-mutant OPN. 


\section{Serum OPN and Clinicopathologic Data}

Using the median serum OPN of the control participants as a cut-off, we assigned the patients to two groups: $36 \mathrm{pa}$ tients $(31.3 \%)$ with low serum OPN ( $\leq 15 \mathrm{ng} / \mathrm{dL})$, and 79 patients $(68.7 \%)$ with high serum OPN (>15 ng/dL).

Older patients more frequently presented with high serum OPN: 45 patients (57\%) in the high OPN group compared with 34 patients (43\%) in the low opN group were 50 or more years of age, and 23 patients $(63.9 \%)$ less than 50 years of age compared with 13 patients $(36.1 \%) 50$ or more years of age had low serum OPN $(p=0.03)$. Patients who were postmenopausal had a significantly high serum OPN level: of patients with high serum OPN, 58 (73.4\%) were postmenopausal and $21(26.6 \%)$ were premenopausal ( $p=$ $0.014)$. However, no significant association of serum OPN with site of metastasis or Ki-67 index was found (Table I).

Our study demonstrated that serum OPN was significantly high ( $p=0.017)$ in patients with HER2-amplified BCa $(64.4 \pm 42.3 \mathrm{ng} / \mathrm{dL})$, followed by triple-negative BCa (55.9 $\pm 34.7 \mathrm{ng} / \mathrm{dL}$ ), and then by the luminal B and A subtypes $(38.4 \pm 33.1 \mathrm{ng} / \mathrm{dL}$ and $36.3 \pm 32.2 \mathrm{ng} / \mathrm{dL}$ respectively)

\section{Polymorphism of OPN and Clinicopathologic Data}

Mutated opN was more frequent in older patients: 43 patients (55.8\%) 50 or more years of age compared with 34 patients $(44.2 \%)$ less than 50 years of age had mutated OPN, and of patients with non-mutated oPN, 23 (60.5\%) were less than 50 years of age and $15(39.5 \%)$ were 50 or more years of age, a difference that was nonsignificant $(p=0.09$ ). We found that mutant OPN was more frequent in postmenopausal patients: mutant OPN was found in 54 postmenopausal patients $(70.1 \%)$ and in 23 premenopausal patients $(29.9 \%)$. Non-mutant OPN was found in 22 postmenopausal patients (57.9\%) compared with 16 premenopausal patients (42.1\%), a difference that was nonsignificant ( $p=0.2$, Table II).

Non-mutant OPN was significantly associated with the presence of visceral metastasis only: of patients with non-mutant OPN, 30 (78.9\%) presented with visceral metastasis only compared with 8 patients $(21.1 \%$ ) who presented with both visceral and bone metastasis. Of patients with mutant OPN, 44 (57.1\%) presented with visceral metastasis

TABLE I Clinicopathologic data by serum osteopontin (OPN) level

\begin{tabular}{|c|c|c|c|}
\hline \multirow[t]{2}{*}{ Variable } & \multicolumn{2}{|c|}{ OPN group $[n(\%)]$} & \multirow{2}{*}{$\begin{array}{c}p \\
\text { Value }\end{array}$} \\
\hline & $\leq 15 \mathrm{ng} / \mathrm{dL}$ & $>15 \mathrm{ng} / \mathrm{dL}$ & \\
\hline \multicolumn{4}{|l|}{ Age } \\
\hline$<50$ Years & $23(63.9)$ & $34(43)$ & \multirow{2}{*}{0.03} \\
\hline$\geq 50$ Years & $13(36.1)$ & $45(57)$ & \\
\hline \multicolumn{4}{|l|}{ Menopausal status } \\
\hline Pre & $18(50)$ & $21(26.6)$ & \multirow{2}{*}{0.014} \\
\hline Post & $18(50)$ & $58(73.4)$ & \\
\hline \multicolumn{4}{|l|}{ Metastasis } \\
\hline Viscera and bone & $8(22.2)$ & 33 (41.8) & \multirow{2}{*}{0.058} \\
\hline Viscera only & $28(77.8)$ & $46(58.2)$ & \\
\hline \multicolumn{4}{|l|}{ Ki-67 index } \\
\hline$\leq 10$ & $17(47.2)$ & $29(36.7)$ & \multirow[t]{2}{*}{0.2} \\
\hline$>10$ & $19(52.8)$ & $50(63.3)$ & \\
\hline
\end{tabular}

only compared with 33 (42.9\%) who presented with both visceral and bone metastasis $(p=0.024)$. Patients with mutated opN were more likely to have a high Ki-67 index: of patients with mutated opN, 51 (66.2\%) had a high Ki-67 index and 26 (33.8\%) had a low Ki-67 index; and of patients with non-mutated OPN, 20 (52.6\%) had a low Ki-67 index and 18 (47.4\%) had a high Ki-67 index, a difference that was nonsignificant ( $p=0.06$, Table II).

By PCR sequencing, OPN gene mutation was found to be remarkably high in patients with triple-negative BCa ( $85 \%$ mutated OPN vs. $15 \%$ non-mutated OPN) and with HER2-overexpressed BCa ( $80 \%$ mutated OPN vs. $20 \%$ non-mutated OPN), followed by luminal B вCa $(61.9 \% \mathrm{mu}-$ tated OPN vs. $38.1 \%$ non-mutated OPN) and luminal A вСа (57.9\% mutated OPN vs. $42.1 \%$ non-mutated OPN).

\section{Serum OPN and OPN Polymorphism and Tumour Response}

Serum OPN was significantly high $(p=0.05)$ in patients who experienced a poor response to first-line chemotherapy: $49.1 \pm 33.8 \mathrm{ng} / \mathrm{dL}$ in patients with progressive disease compared with $35.5 \pm 34.3 \mathrm{ng} / \mathrm{dL}$ in those who achieved a complete response, a partial response, or stable disease (Table III).

Patients with mutated OPN by PCR sequencing also showed a significantly poor response. Of patients with mutant OPN, $54(70.1 \%)$ developed disease progression and $23(29.9 \%)$ achieved either a complete response, a partial response, or stable disease; however, $50 \%$ of patients with non-mutated OPN experienced disease progression $(p=0.04)$.

TABLE II Clinicopathologic data by osteopontin (OPN) polymorphism

\begin{tabular}{lccc}
\hline \multicolumn{1}{c}{ Variable } & \multicolumn{2}{c}{ OPN group [n (\%)] } & $\begin{array}{c}\boldsymbol{p} \\
\text { Value }\end{array}$ \\
\cline { 2 - 3 } & Non-mutated & Mutated & \\
\hline Age & & & \\
$\quad<50$ Years & $23(60.5)$ & $34(44.2)$ & 0.09 \\
$\quad \geq 50$ Years & $15(39.5)$ & $43(55.8)$ & \\
Menopausal status & & & \\
$\quad$ Pre & $16(42.1)$ & $23(29.9)$ & 0.2 \\
$\quad$ Post & $22(57.9)$ & $54(70.1)$ & \\
Metastasis & & & \\
$\quad$ Viscera and bone & $8(21.1)$ & $33(42.9)$ & 0.024 \\
$\quad$ Viscera only & $30(78.9)$ & $44(57.12)$ & \\
Ki-67 index & & & \\
$\quad \leq 10$ & $20(52.6)$ & $26(33.8)$ & 0.06 \\
$>10$ & $18(47.4)$ & $51(66.2)$ & \\
\hline
\end{tabular}

TABLE III Tumour response in patients with serum osteopontin (OPN) and OPN polymorphism

\begin{tabular}{lcccc}
\hline \multicolumn{1}{|c}{ Response type } & $\begin{array}{c}\text { Pts } \\
(\boldsymbol{n})\end{array}$ & $\begin{array}{c}\text { Mean OPN } \\
(\mathbf{n g} / \mathbf{d L})\end{array}$ & $\boldsymbol{t}$ & $\begin{array}{c}\boldsymbol{p} \\
\text { Value }\end{array}$ \\
\hline CR, PR, and SD & 41 & $35.5 \pm 34.3$ & & \\
\hline Progressive disease & 73 & $49.1 \pm 33.8$ & 1.9 & 0.05 \\
\hline
\end{tabular}

Pts = patients; $\mathrm{CR}=$ complete response; $\mathrm{PR}=$ partial response; $\mathrm{SD}=$ stable disease. 


\section{Serum OPN and OPN Polymorphism and Patient Survival}

In patients with both high serum OPN and mutated OPN, PFS was significantly poor (median: 12 months vs. 14 months, $p=0.001$, at a median follow-up duration of 15 months). Those patients also experienced significantly poor os (median: 14 months vs. 18 months, $p=0.001$ ), as shown in Table IV and Figure 1.

TABLE IV Patient survival by serum osteopontin (OPN) level and OPN polymorphism

\begin{tabular}{|c|c|c|c|c|c|c|}
\hline \multirow[t]{2}{*}{ Survival type } & \multicolumn{6}{|c|}{ OPN group } \\
\hline & $\begin{array}{c}\leq 15 \mathrm{ng} / \mathrm{dL} \\
(n=36)\end{array}$ & $\begin{array}{c}>15 \mathrm{ng} / \mathrm{dL} \\
(n=79)\end{array}$ & $\begin{array}{c}p \\
\text { Value }\end{array}$ & $\begin{array}{c}\text { Non-mutated } \\
(n=38)\end{array}$ & $\begin{array}{c}\text { Mutated } \\
(n=77)\end{array}$ & $\begin{array}{c}p \\
\text { Value }\end{array}$ \\
\hline \multicolumn{7}{|l|}{ Progression-free (months) } \\
\hline Median & 14 & 12 & 0.001 & 14 & 12 & 0.001 \\
\hline $95 \% \mathrm{Cl}$ & 12.7 to 15.2 & 11.5 to 12.5 & & 13.1 to 14.8 & 11.4 to 12.5 & \\
\hline \multicolumn{7}{|l|}{ Overall (months) } \\
\hline Median & 18 & 14 & 0.001 & 18 & 14 & 0.001 \\
\hline $95 \% \mathrm{Cl}$ & 15.8 to 20.1 & 12.9 to 15.01 & & 15.1 to 20.8 & 12.6 to 15.3 & \\
\hline
\end{tabular}

$\mathrm{Cl}=$ confidence interval.
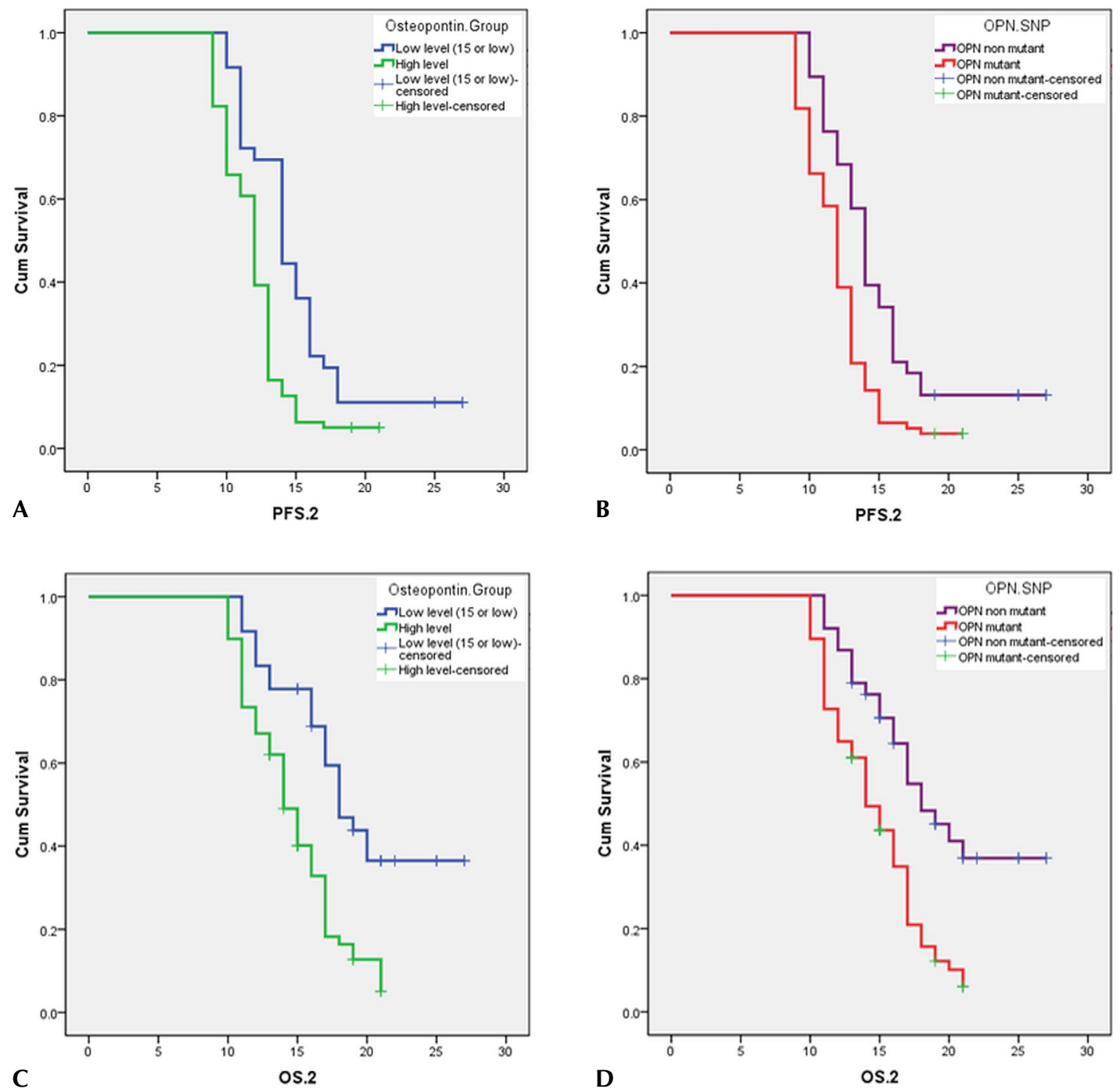

FIGURE 1 Serum osteopontin (OPN) and osteopontin polymorphism relative to patient survival. (A) Progression-free survival (PFS) by OPN level. (B) Overall survival (OS) by OPN level. (C) PFS by OPN without or with single-nucleotide polymorphism. (D) OS by OPN without or with singlenucleotide polymorphism. 


\section{Concordance}

Complete concordance between high serum OPN as detected by ELISA and OPN polymorphism as detected by PCR sequencing was observed in 69 tumours (79.3\%). Complete concordance between low serum OPN and non-mutated OPN was observed in 28 tumours (60.8\%, Figure 2$)$.

\section{DISCUSSION}

Osteopontin is considered to be an integrin-binding phosphoprotein, and it is found in normal cells ${ }^{11}$. It has an important role in many malignancies through its involvement in both tissue invasion and metastasis ${ }^{12,13}$, and it has been correlated with aggressiveness in many cancers, including BCa. In an investigation conducted by Cook et al. ${ }^{14}$, oPN gene expression was detected in a human model of BCa. The alterations caused by OPN expression represent the 6 "cancer hallmarks" in a BCa progression model. Crosstalk between oPN and many intracellular signalling pathways such as Akt, Raf/MEK/ERK, ILK/PI3K/GSK3B ${ }^{15}$, and Ran GTPase/c-Met/PI3K is worth noting ${ }^{16}$.

Resistance to chemotherapy is an important cause of treatment failure. Many studies indicate that drug resistance can be influenced by factors such as chemokines, cytokines, cell adhesion molecules, and growth factors in the cancer microenvironment. Previous reports showed that OPN provokes non-classical triggering of the Hedgehog signalling pathway, stimulates translocation of the GLI1 "transcription factor" in the nucleus, and produces GLI1dependent overexpression of multidrug resistance proteins $A B C G 2$ and $A B C B 1{ }^{17}$. Cytokine signalling of $A B C$ protein expression in $\mathrm{BCa}$ was linked to chemotherapy resistance. In cell culture, oPN causes resistance to cyclophosphamide ${ }^{18}$, doxorubicin $^{19}$, and paclitaxel and cisplatin ${ }^{17}$ in BCa, which is attributed to well-defined antiapoptotic properties or capability to upregulate drug exporters.

High OPN in primary tumours was found to be associated with poor prognosis and advanced-stage disease ${ }^{20}$. Also, OPN can be detected in the blood of patients with various cancers including those of colon, prostate, and stomach; bronchogenic carcinoma; and hepatocellular carcinoma $^{21,22}$.

\section{BCa Subtypes}

An interesting result in the present study is the significantly high serum OPN and increase in mutated OPN in the HER2-amplified and triple-negative BCa subtypes, followed by the luminal B and A subtypes. In contrast, Anborgh

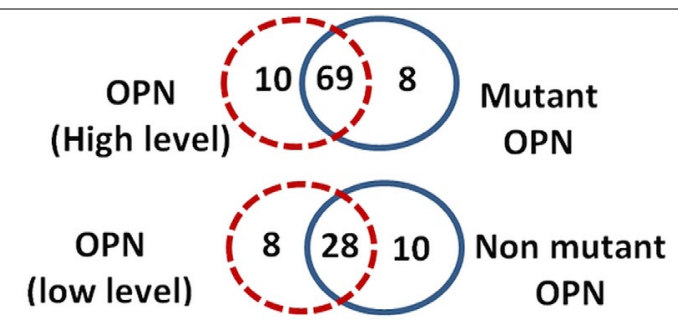

FIGURE 2 Concordance between serum osteopontin (OPN) and OPN gene polymorphism. et al. ${ }^{22}$ did not find any association between serum OPN and the BCa subtypes, which might be explained by the fact that the patients included in our study had metastatic disease, while the Anborgh etal. study included patients with locally advanced disease. Alternatively, the discrepancy might be related to the different demographic characteristics of the patients in both studies. Our data might open the way to a prognostic model or the development of an OPN targeting molecule for the triple-negative BCa subtype, which is an aggressive disease that lacks targeted agents.

\section{Response}

With respect to the tumour response in our cohort, patients with either mutated OPN or high serum oPN had a poor response to chemotherapy or showed more drug resistance, and so a pretreatment OPN assessment could be considered for tailoring and customizing therapy for metastatic вCa. Our result is supported by Bramwell et al..$^{23}$, who reported a high level of OPN in most patients with metastatic BCa that was linked with poor tumour response and high disease burden.

Moreover, frequent measurement by Singal and colleague ${ }^{23,24}$ of serum OPN in patients with metastatic BCa revealed decreased survival (despite treatment) as serum OPN increased over time. Serum opN assessment might therefore possess prognostic and predictive value in advanced BCa. In another report involving patients with locally advanced BCa, which compared pretreatment serum oPN with the pathologic response after neoadjuvant therapy, the authors found a higher median serum OPN level in patients with a poor response than in patients who attained a pathologic complete response ${ }^{25}$, which accords with the results in our study.

According to two in vitro studies conducted in a $\mathrm{BCa}$ model cell line, suppression of OPN gene expression can promote cell apoptosis and ameliorate radiosensitivity, suggesting that OPN might be a unique target for enhancement of radiotherapy ${ }^{26,27}$.

\section{Survival}

Our report confirms OPN as a prognostic marker in metastatic BCa, given our finding that patients with increased serum OPN and OPN gene polymorphism experienced the same significantly poorer PFS (median: 12 months vs. 14 months, $p=0.001$ ) and os (median: 14 months vs. 18 months, $p=0.001$ ). Those results are consistent with findings in an earlier cohort of 70 patients with confirmed metastatic BCa, in which $70 \%$ showed increased serum oPN and significantly shorter survival times ${ }^{24}$.

Bramwell et al. ${ }^{23}$ conducted a larger study of patients with metastatic $\mathrm{BCa}$, reporting elevated pretreatment serum OPN in $66 \%$ of the patients, which was inversely and significantly correlated with survival. Furthermore, consecutive measurements of serum oPN demonstrated that, in that cohort, an increase of serum opN at any time was highly prognostic for poor survival. Importantly, an obvious correlation between baseline serum opN and patient survival was evident. Another study found that, compared with patients having a baseline plasma opN level below the median, those with a level above the median were significantly more likely to die from disease progression (hazard ratio: $3.4 ; 95 \%$ 
confidence interval: 1.4 to $11.3 ; p=0.011$ ) and that baseline plasma OPN was significantly associated with poor survival $(p=0.002)^{25}$. Using an ELISA assay, another study showed that, in women with metastatic BCa, compared with healthy women or patients who had received definitive treatment for primary $\mathrm{BCa}$, plasma OPN was elevated and associated with poor survival ${ }^{24}$.

Authors studying a cohort of 154 women with lymph-node-negative BCa stated that expression of OPN in tumour cells (immunopositivity), by various methods of assessment and a semi-quantitative immunohistochemistry scoring system, was significantly correlated with both decreased disease-free survival and ${ }^{28}{ }^{28}$. Likewise, Tuck et al. and Rudland et al. ${ }^{29,30}$ reported a significant association between OPN positivity and a poor prognosis in early BCa, even when a semi-quantitative immunohistochemistry scoring system was used. Hence, oPN represents the first well-known prognostic tumour marker for os in patients with either locally advanced or metastatic BCa. In comparison, OPN evaluation in tumour cells and plasma from a particular subtype of hormone-sensitive early вса typically showed better prognosis ${ }^{21}$. Interestingly, neither plasma nor tumour OPN had yet been used to provide prognostic information in any multivariate research. The authors therefore concluded that tumour or plasma OPN in postmenopausal hormone-sensitive early BCa might have minimal prognostic value ${ }^{21}$. That finding might be explained by differences in the included patients. Our study considered patients with metastatic BCa of all subtypes, including HER2-amplified and triple-negative; however, the former study included only patients with hormone-sensitive early BCa that actually might have a good prognosis.

To the best of our knowledge, our study is the first to confirm an association between two validated techniques for biologic profile assessment in patients with cancer. We consider OPN to be a significant prognostic and treatment tailoring marker in metastatic $\mathrm{BCa}$, given that concordance between high serum OPN by ELISA and OPN gene mutation by PCR sequencing was found in 69 tumours (79.3\%) and that 28 tumours showed complete concordance between low serum OPN by ELISA and non-mutated OPN (60.8\%). Based on those results, either technique could be considered reliable for opN detection depending on the facilities available in a cancer centre.

\section{CONCLUSIONS}

This prospective study demonstrates a statistically significant relation of response and survival in patients with BCa and pretreatment serum OPN or OPN gene mutation. High baseline serum OPN and identification of OPN gene mutation were both correlated with poor outcome, suggesting that OPN could be proposed as a biomarker for BCa progression. Moreover, concordance between the ELISA and PCR sequencing techniques was evident, and hence, either approach could be used to test OPN. Mutation of the oPN gene was frequently found in the triple-negative BCa subtype (one of the most aggressive subtypes of BCa, which lacks targeted therapies), and so in future studies, OPN mutation might be used to tailor chemotherapy treatment or OPN could be considered a target molecule for new therapies.

\section{CONFLICT OF INTEREST DISCLOSURES}

We have read and understood Current Oncology's policy on disclosing conflicts of interest, and we declare that we have none.

\section{AUTHOR AFFILIATIONS}

*Medical Oncology Unit, Oncology Center, Mansoura Faculty of Medicine; ${ }^{\dagger}$ Clinical Pathology Department, Mansoura University; ${ }^{\ddagger}$ Clinical Oncology and Nuclear Medicine Department, and ${ }^{\S}$ Hematology Unit, Clinical Pathology Department, Mansoura University, Mansoura, Egypt.

\section{REFERENCES}

1. Shevde LA, Samant RS. Role of osteopontin in the pathophysiology of cancer. Matrix Biol 2014;37:131-41.

2. Denhardt DT, Mistretta D, Chambers AF, et al. Transcriptional regulation of osteopontin and the metastatic phenotype: evidence for a Ras-activated enhancer in the human OPN promoter. Clin Exp Metastasis 2003;20:77-84.

3. Atai NA, Bansal M, Lo C, et al. Osteopontin is up-regulated and associated with neutrophil and macrophage infiltration in glioblastoma. Immunology 2011;132:39-48.

4. Štemberger C, Matusan-Ilifas K, Avirovic M, et al. Osteopontin is associated with decreased apoptosis and $\alpha \mathrm{v}$ integrin expression in lung adenocarcinoma. Acta Histochem 2014; 116:222-9.

5. Sun BS, You J, Li Y, Zhang ZF, Wang CL. Osteopontin knockdown suppresses non-small cell lung cancer cell invasion and metastasis. Chin Med J (Engl) 2013;126:1683-8.

6. Ramachandran S, Kwon KY, Shin SJ, et al. Regulatory role of osteopontin in malignant transformation of endometrial cancer. Mol Biol Rep 2013;40:3623-9.

7. KumarV, Behera R, Lohite K, Karnik S, Kundu GC. p38 Kinase is crucial for osteopontin-induced furin expression that supports cervical cancer progression. Cancer Res 2010;70:10381-91.

8. Allan AL, George R, Vantyghem SA, et al. Role of the integrinbinding protein osteopontin in lymphatic metastasis of breast cancer. Am J Pathol 2006;169:233-46.

9. Chakraborty G, Jain S, Kundu GC. Osteopontin promotes vascular endothelial growth factor-dependent breast tumor growth and angiogenesis via autocrine and paracrine mechanisms. Cancer Res 2008;68:152-61.

10. Das R, Mahabeleshwar GH, Kundu GC. Osteopontin induces AP-1-mediated secretion of urokinase type plasminogen activator through c-Src dependent EGF receptor transactivation in breast cancer cells. J Biol Chem 2004;279:11051-64.

11. Rangaswami H, Bulbule A, Kundu GC. Osteopontin: role in cell signaling and cancer progression. Trends Cell Biol 2006; 16:79-87.

12. Anborgh PH, Mutrie JC, Tuck AB, Chambers AF. Role of the metastasis-promoting protein osteopontin in the tumour microenvironment. J Cell Mol Med 2010;14:2037-44.

13. Tuck AB, Chambers AF, Allan AL. Osteopontin overexpression in breast cancer: knowledge gained and possible implications for clinical management. J Cell Biochem 2007;102:859-68.

14. Cook AC, Tuck AB, McCarthy S, et al. Osteopontin induces multiple changes in gene expression that reflect the six "hallmarks of cancer" in a model of breast cancer progression. Mol Carcinog 2005;43:225-36.

15. Robertson BW, Bonsal L, Chellaiah MA. Regulation of Erk1/2 activation by osteopontin in PC3 human prostate cancer cells. Mol Cancer 2010;9:260.

16. Yuen HF, Chan KK, Grills C, et al. Ran is a potential therapeutic target for cancer cells with molecular changes associated with activation of the PI3K/Akt/mTORC1 and Ras/MEK/ERK pathways. Clin Cancer Res 2012;18:380-91.

17. Das S, Samant RS, Shevde LA. Nonclassical activation of Hedgehog signaling enhances multidrug resistance and 
makes cancer cells refractory to SMOH-targeting Hedgehog inhibition. J Biol Chem 2013;288:11824-33.

18. Pang H, Cai L, Yang Y, Chen X, Sui G, Zhao C. Knockdown of osteopontin chemosensitizes MDA-MB-231 cells to cyclophosphamide by enhancing apoptosis through activating p38 MAPK pathway. Cancer Biother Radiopharm 2011;26:165-73.

19. Yang L, Wei L, Zhao W, et al. Down-regulation of osteopontin expression by RNA interference affects cell proliferation and chemotherapy sensitivity of breast cancer MDA-MB-231 cells. Mol Med Rep 2012;5:373-6.

20. Coppola D, Szabo M, Boulware D, et al. Correlation of osteopontin protein expression and pathological stage across a wide variety of tumor histologies. Clin Cancer Res 2004;10:184-90.

21. Bramwell VHC, Tuck AB, Chapman JAW, et al. Assessment of osteopontin in early breast cancer: correlative study in a randomised clinical trial. Breast Cancer Res 2014;16:R8.

22. Anborgh PH, Wilson SM, Tuck AB, et al. New dual monoclonal ELISA for measuring plasma osteopontin as a biomarker associated with survival in prostate cancer: clinical validation and comparison of multiple ELISAs. Clin Chem 2009;55:895-903.

23. Bramwell VH, Doig GS, TuckAB, et al. Serial plasma osteopontin levels have prognostic value in metastatic breast cancer. Clin Cancer Res 2006;12:3337-43.
24. Singhal H, Bautista DS, Tonkin KS, et al. Elevated plasma osteopontin in metastatic breast cancer associated with increased tumor burden and decreased survival. Clin Cancer Res 1997;3:605-11.

25. Anborgh PH, Caria LB, Chambers AF, et al. Role of plasma osteopontin as a biomarker in locally advanced breast cancer. Am J Transl Res 2015;7:723-32.

26. Yang L, Zhao W, Zuo WS, et al. Silencing of osteopontin promotes the radiosensitivity of breast cancer cells by reducing the expression of hypoxia inducible factor 1 and vascular endothelial growth factor. Chin Med J (Engl) 2012;125:293-9.

27. Hahnel A, Wichmann H, Kappler M, et al. Effects of osteopontin inhibition on radiosensitivity of MDA-MB-231 breast cancer cells. Radiat Oncol 2010;5:82.

28. Tuck AB, O'Malley FP, Harris JF, et al. Osteopontin expression in a group of lymph node negative breast cancer patients. Int J Cancer 1998;79:502-8.

29. Wang G, Platt-Higgins A, Carroll J, et al. Induction of metastasis by S100P in a rat mammary model and its association with poor survival of breast cancer patients. Cancer Res 2006;66:1199-207.

30. Rudland PS, Platt-Higgins A, El-Tanani M, et al. Prognostic significance of the metastasis-associated protein osteopontin in human breast cancer. Cancer Res 2002;62:3417-27. 